\title{
Weighted Cheeger constant and first eigenvalue lower bound estimates on smooth metric measure spaces
}

\author{
Abimbola Abolarinwa 1* ${ }^{1}$, Akram Ali $^{2}$ and Ali Alkhadi²
}

\section{"Correspondence:}

a.abolarinwa1@gmail.com

'Department of Mathematics,

University of Lagos, Akoka, Lagos

State, Nigeria

Full list of author information is

available at the end of the article

\section{Springer}

\begin{abstract}
We establish new eigenvalue inequalities in terms of the weighted Cheeger constant for drifting $p$-Laplacian on smooth metric measure spaces with or without boundary. The weighted Cheeger constant is bounded from below by a geometric constant involving the divergence of suitable vector fields. On the other hand, we establish a weighted form of Escobar-Lichnerowicz-Reilly lower bound estimates on the first nonzero eigenvalue of the drifting bi-Laplacian on weighted manifolds. As an application, we prove buckling eigenvalue lower bound estimates, first, on the weighted geodesic balls and then on submanifolds having bounded weighted mean curvature.
\end{abstract}

MSC: Primary 22E30; secondary 26D10; 35P30; 58J05

Keywords: Cheeger constant; Drifting Laplacian; Eigenvalues; Riemannian manifold; Mean curvature

\section{Introduction}

The first aim of this paper is to answer in the affirmative that there exists a weighted form of Cheeger constant for domains in complete smooth metric measure spaces. The determination of Cheeger constant on a domain aids the process of solving isoperimetric problems (see [14, 27, 33, 37] for examples) and the classical Cheeger problems (see [31] and the references therein). Here we show that the weighted Cheeger constant is bounded from below by some other geometric constant involving the divergence of suitable vector fields. Our main result (Theorem 2.3) is the generalized Cheeger inequality, which states that the first nonzero eigenvalue of the drifting $p$-Laplacian $(1<p<\infty)$ on a bounded domain is bounded from below by a multiple of Cheeger constant depending on $p$.

In the second part of the paper (Sect. 3), we establish a weighted form of EscobarLichnerowicz-Reilly lower bound estimates on the first nonzero eigenvalue of the drifting bi-Laplacian defining buckling problem on weighted manifolds with generalized Ricci curvature bounded from below by a nonnegative constant. This result is a generalization of $[17,38]$ in the case of bounded domains with smooth boundary in Riemannian manifolds. See also similar results $[26,35,36,39]$ for the $p$-Laplacian or drifting Laplacian eigenvalue

(c) The Author(s) 2021. This article is licensed under a Creative Commons Attribution 4.0 International License, which permits use sharing, adaptation, distribution and reproduction in any medium or format, as long as you give appropriate credit to the original author(s) and the source, provide a link to the Creative Commons licence, and indicate if changes were made. The images or other third party material in this article are included in the article's Creative Commons licence, unless indicated otherwise in a credit line to the material. If material is not included in the article's Creative Commons licence and your intended use is not permitted by statutory regulation or exceeds the permitted use, you will need to obtain permission directly from the copyright holder. To view a copy of this licence, visit http://creativecommons.org/licenses/by/4.0/. 
problems. For applications, following closely the idea of Hessian comparison estimates applied in $[11,19,25]$ on different problems, we discuss lower bound estimates for the first eigenvalues on the weighted geodesic ball whose radius does not exceed the injectivity radius and submanifolds having bounded weighted mean curvature. In fact, our estimates extend the results of $[11,25,38]$ when the supremum of the sectional curvature inside the ball is nonnegative.

The rest of this section is devoted to giving basic information (that is relevant to our study) about smooth metric measure spaces. We also make some remarks on previous relevant results on eigenvalues in this class of spaces before this section is concluded.

Smooth metric measure spaces Let $\mathscr{M}$ be an $n$-dimensional complete manifold endowed with Riemannian metric $\mathrm{g}$ and volume measure $d v$. Given a smooth function $\phi: \mathscr{M} \rightarrow \mathbb{R}$, the triple $(\mathscr{M}, \mathrm{g}, \phi)$ is referred to as a complete smooth metric measure space, where the weighted measure $e^{-\phi} d v$ is conformally related to the Riemannian volume measure $d v$.

With $(\mathscr{M}, \mathrm{g}, \phi)$ we associate the generalized Ricci curvature known as the Bakry-Émery curvature defined by

$$
\operatorname{Ric}_{\phi}^{m}:=\operatorname{Ric}_{\mathrm{g}}+\nabla \nabla \phi-\frac{\nabla \phi \otimes \nabla \phi}{m-n}, \quad m \geq n,
$$

where $\mathrm{Ric}_{\mathrm{g}}$ and $\nabla$ respectively denote the Ricci tensor and gradient operator with respect to the Riemannian manifold $(\mathscr{M}, \mathrm{g})$, and $\nabla \nabla \phi$ is the Hessian of $\phi$. Note that the case $m=n$ is attainable only for a constant function $\phi$, and then $\operatorname{Ric}_{\phi}^{m} \equiv \operatorname{Ric}_{\mathrm{g}}$. We obtain $\operatorname{Ric}_{\phi}^{\infty}:=\operatorname{Ric}_{\mathrm{g}}+$ $\nabla \nabla \phi$ as $m$ approaches $\infty$. The generalized Ricci curvature carries significant information for various $m$, for instance, $\operatorname{Ric}_{\phi}=\alpha \mathrm{g}, \alpha \in \mathbb{R}$, defines gradient Ricci solitons as singularity formations for the Ricci flow [22].

Also, we associate with $(\mathscr{M}, \mathrm{g}, \phi)$ a self-adjoint second-order differential operator, called the drifting Laplacian (or Witten, or weighted Laplacian), which is defined for any smooth function $u$ on $\mathscr{M}$ by

$$
\mathscr{L}_{\phi} u:=e^{\phi} \operatorname{div}\left(e^{-\phi} \nabla \phi\right)=\Delta u-\langle\nabla \phi, \nabla u\rangle,
$$

where $\Delta$ and $\langle\cdot, \cdot\rangle$ respectively stand for the Laplace-Beltrami operator and Riemannian inner product on $(\mathscr{M}, \mathrm{g})$.

Clearly, a smooth metric measure space is a natural extension of a Riemannian manifold, whereas the drifting Laplacian and Bakry-Émery tensor (generalized Ricci curvature) are the natural counterparts for the Laplace-Beltrami operator and the Ricci curvature, respectively. Smooth metric measure spaces play a central role throughout geometric analysis, probability theory, quantum field theory, and statistical mechanics and have close links with Markov diffusion operators, generalized curvature, and geometry [8, 9]. A striking application of this class of spaces is already seen in Perelman's approach to resolving the Poincaré and geometrization conjectures [32]. See also [29, 30].

Estimating eigenvalues of a prescribed elliptic operator, such as the Laplacian, $p$ Laplacian, drifting Laplacian, and the biharmonic operator, has been an intensive research area because of its relevance in geometry and physics. Many classical results around this research area are well captured in the monographs [14, 21,33]. Recently, several authors have engaged in finding lower and upper geometric estimates for the first eigenvalues of 
the above listed Laplace-type operators with several geometric quantities formulated and analyzed. For example, Futaki, $\mathrm{Li}$, and $\mathrm{Li}$ [20] obtained a lower bound on the first eigenvalue of drifting Laplacian on a compact Riemannian manifold without boundary under the assumption that the generalized Ricci curvature is bounded from below. The lower bound obtained depends on the diameter of the manifold and extends the previous result by Shi and Zhang [34] for the Laplacian. As an application, we derive a lower bound estimate for the diameter of compact gradient shrinking Ricci soliton.

Inspired by this, Wang and Li [36] combined weighted $p$-Bochner and $p$-Reilly formulas with gradient estimate technique to derive lower bound of Escobar-Lichnerowicz-Reilly type on the first eigenvalue of drifting $p$-Laplacian on a compact smooth metric measure space $M$ in terms of the sign of the Bakry-Émery Ricci curvature. Namely, for $p \geq 2$, $\operatorname{Ric}_{\phi}^{m} \geq k g, k>0$, and the first eigenvalue $\lambda_{1, p}^{\partial M}$ of $-\mathscr{L}_{\phi, p}$ on $M$, we have

$$
\lambda_{1, p}^{\partial M} \geq \frac{1}{(1-p)^{p-1}}\left(\frac{m k}{m-1}\right)^{\frac{p}{2}}
$$

where $\lambda_{1, p}^{\partial M}=\lambda_{1, p}$ if $\partial M=\varnothing, \lambda_{1, p}^{\partial M}=\lambda_{1, p}^{\text {Dir }}$ if $\partial M \neq \varnothing$ but with Dirichlet boundary condition, and $\lambda_{1, p}^{\partial M}=\lambda_{1, p}^{\mathrm{Neu}}$ if $\partial M \neq \varnothing$ but with Neumann boundary condition. Moreover, for the Dirichlet eigenvalue, the weighted mean curvature must be nonnegative, whereas the boundary is assumed to be convex for the Neumann eigenvalue.

Recall that the drifting Laplacian is an important self-adjoint elliptic operator associated with the Ricci soliton from the Ricci flow theory and self-shrinker from the mean curvature flow theory. Therefore estimating eigenvalue inequalities for the drifting Laplacian has natural consequences on both flows. Zeng [39] considered the eigenvalue problem for the bi-drifting Laplacian describing the buckling problem on a bounded domain of the complete smooth metric measure space and obtained a general inequality of Ashbaugh-Cheng-Ichikawa-Mamestsuka type on a cigar metric measure space and Gaussian shrinking Ricci soliton under some restrictive conditions. In [24] (see also [40]) the authors studied a clamped plate eigenvalue problem for the bi-drifting Laplacian on a bounded domain of a cigar soliton and then derive corresponding universal inequalities. The steady gradient Ricci soliton emanated from the Hamilton Ricci flow [22,32] is a cigar soliton in dimension two. A cigar soliton also appears as the Euclidean-Witten black hole under first-order renormalization group flow of the world-sheet sigmal model [23] in general relativity theory. Thus the research in [24,39], and [40] is very important and interesting in both geometry and physics. Related issues $[3,5,7,32]$ are discussed under evolving geometry.

On the other hand, problems of finding the Cheeger constant are very interesting with several geometric and physical applications; see $[1,4,10,31]$ and the references therein. Especially, bounding the first eigenvalue from below by the Cheeger constant has yielded several interesting results [27] since the pioneering work [15]. Note that the weighted Cheeger constant introduced here (Definition 2.2) is new and clearly extends the classical one to the setting of weighted measure. This hopefully will help tackle isoperimetric problems on weighted manifolds. 


\section{Weighted Cheeger constant and drifting $p$-Laplacian}

\subsection{First eigenvalue of the drifting $p$-Laplacian}

The drifting $p$-Laplace operator for $p \in(1, \infty)$ is defined by

$$
\mathscr{L}_{\phi, p} f:=e^{\phi} \operatorname{div}\left(e^{-\phi}\|\nabla f\|^{p-2} \nabla f\right)=\mathbb{L}_{p} f-\|\nabla f\|^{p-2}\langle\nabla \phi, \nabla f\rangle,
$$

where $\mathbb{L}_{p} f=\operatorname{div}\left(\|\nabla f\|^{p-2} \nabla f\right)$ is the usual $p$-Laplacian of $f$, which coincides with $\mathscr{L}_{\phi, p}$ when $\phi$ is a constant. The case where $p=2$ implies that $\mathscr{L}_{\phi, p}$ is the drifting Laplacian $\mathscr{L}_{\phi}$ and $\mathbb{L}_{p}$ is the usual Laplacian $\Delta$. For a compact weighted Riemannian manifold $M \subset(\mathscr{M}, \mathrm{g}, \phi)$ with smooth boundary, we consider the nonlinear eigenvalue problem (for finding $\lambda_{p}$ and nonzero $f$ )

$$
-\mathscr{L}_{\phi, p} f=\lambda_{p}|f|^{p-2} f \quad \text { in } M
$$

together with Dirichlet boundary condition

$$
f=0 \quad \text { on } \partial M
$$

or Neumann boundary condition

$$
\frac{\partial f}{\partial v}=0 \quad \text { on } \partial M,
$$

if the boundary is nonempty, where $v$ is the outward unit normal vector field to $\partial M$. Problem (2.2) is understood in the weak sense, meaning that

$$
\int_{M}\|\nabla f\|^{p-2}\langle\nabla f, \nabla \psi\rangle e^{-\phi} d \nu=\lambda_{p} \int_{M}|f|^{p-2} f \psi e^{-\phi} d \nu .
$$

for $f \in W_{0}^{1, p}(M)$ and every test function $\psi \in C_{0}^{\infty}(M)$, where $W_{0}^{1, p}(M)$ is the completion of $C_{0}^{\infty}(M)$. The first eigenvalue $\lambda_{1, p}(M)$ of the drifting $p$-Laplacian on $M$ can be characterized by

$$
\lambda_{1, p}(M)=\inf _{f}\left\{\frac{\int_{M}\|\nabla f\|^{p} e^{-\phi} d \nu}{\int_{M}|f|^{p} e^{-\phi} d \nu}: f \in W_{0}^{1, p}(M), f \neq 0\right\}
$$

with the constraint $\int_{M}|f|^{p-2} f e^{-\phi} d v=0$.

\subsection{Weighted Cheeger constant}

Definition 2.1 ([6]) Let $(\mathscr{M}, \mathrm{g}, \phi)$ be a smooth metric measure space. For a domain $\Omega \subset$ $\mathscr{M}$, we denote by $\mathscr{X}(\Omega)$ the set of all smooth vector fields $V$ on $\Omega$ satisfying the properties $\|V\|_{\infty}=\sup _{\Omega}\|V\|<\infty$ and $\inf _{\Omega}\left[e^{\phi} \operatorname{div}\left(e^{-\phi} V\right)\right]>0$. Then we define the Cheeger constant as follows:

$$
h(\Omega):=\sup _{V}\left\{\frac{\inf _{\Omega}\left[e^{\phi} \operatorname{div}\left(e^{-\phi} V\right)\right]}{\sup _{\Omega}\|V\|}: V \in \mathscr{X}(\Omega)\right\} .
$$

Evidently, $h$ is nonnegative, and the assignment $\Omega \mapsto h(\Omega)$ satisfies the domain monotonicity property: $\Omega_{1} \subset \Omega_{2} \Longrightarrow h\left(\Omega_{1}\right) \geq h\left(\Omega_{2}\right)$. Moreover, if $\mathscr{X}(\Omega)=\varnothing$, then we set $h(\Omega)=0$, and so clearly $h(\Omega)>0$ if and only if $\mathscr{X}(\Omega) \neq \varnothing$ by $(2.6)$. 
Definition 2.2 Let $(\mathscr{M}, \mathrm{g}, \phi)$ be a smooth metric measure space. The weighted Cheeger constant $\mathbf{h}_{\phi}(\Omega)$ of a domain $\Omega \subset \mathscr{M}$ is defined as

$$
\mathbf{h}_{\phi}(\Omega):=\inf _{\Omega^{\prime}} \frac{\operatorname{Per}_{\phi}\left(\partial \Omega^{\prime}\right)}{\operatorname{Vol}_{\phi}\left(\Omega^{\prime}\right)},
$$

where $\Omega^{\prime}$ ranges over all sufficiently regular $\Omega^{\prime} \Subset \Omega$ with smooth boundary $\partial \Omega^{\prime}$. Here $\mathrm{Vol}_{\phi}$ and $\operatorname{Per}_{\phi}$ stand for the weighted volume and perimeter with respect to the volume and surface measures $e^{-\phi} d v$ and $e^{-\phi} d A$ on $\mathscr{M}$, respectively.

Let us now briefly discuss some upper and lower bounds on $h$. Firstly, by taking any sufficiently regular $E \Subset \Omega$, we can write

$$
\begin{aligned}
\inf _{\Omega}\left[e^{\phi} \operatorname{div}\left(e^{-\phi} V\right)\right] \int_{E} e^{-\phi} d v & \leq \int_{E}\left[e^{\phi} \operatorname{div}\left(e^{-\phi} V\right)\right] e^{-\phi} d v=\int_{\partial E}\langle V, v\rangle e^{-\phi} d A \\
& \leq \int_{\partial E}|\langle V, v\rangle| e^{-\phi} d A \leq \sup _{\Omega}\|V\| \int_{\partial E} e^{-\phi} d A,
\end{aligned}
$$

where $v$ is the outer unit normal vector field on $\partial E$, yielding

$$
\frac{\inf _{\Omega}\left[e^{\phi} \operatorname{div}\left(e^{-\phi} V\right)\right]}{\sup _{\Omega}\|V\|} \leq \frac{\operatorname{Per}_{\phi}(\partial E)}{\operatorname{Vol}_{\phi}(E)}
$$

Now upon taking the supremum on the left over $V \in \mathscr{X}(\Omega)$ and the infimum on the right over $E$, we arrive at an upper bound on $h(\Omega)$ in terms of $\mathbf{h}_{\phi}(\Omega)$, that is,

$$
h(\Omega) \leq \mathbf{h}_{\phi}(\Omega) .
$$

In some particular cases, it may be possible to show that the equality in the last bound is achieved, for instance, in the Euclidean ball setting. The reason for introducing $h(\Omega)$ (see [6]) is to obtain lower bounds for $\lambda_{1, p}(\Omega)$ in terms of any lower bound for $h(\Omega)$. As for a lower bound on $h$, we note that if $\|\nabla \phi\| \leq c$ for some constant $c \geq 0$, then for any vector field $V \in \mathscr{X}(\Omega)$, we have the bound

$$
\inf _{\Omega}\left[e^{\phi} \operatorname{div}\left(e^{-\phi} V\right)\right]=\inf _{\Omega}[\operatorname{div}(V)-\langle\nabla \phi, V\rangle] \geq\left[\inf _{\Omega} \operatorname{div}(V)-c \sup _{\Omega}\|V\|\right]_{+},
$$

where $[\alpha]_{+}:=\max \{\alpha, 0\}$ for $\alpha \in \mathbb{R}$. Hence the lower bound

$$
h(\Omega) \geq h_{c}(\Omega)
$$

can be deduced by quotienting over $\|V\|_{\infty}$ and then taking the supremum over all $V \in$ $\mathscr{X}(\Omega)$, where

$$
h_{c}(\Omega):=\sup _{V}\left\{\frac{\left[\inf _{\Omega} \operatorname{div}(V)-c \sup _{\Omega}\|V\|\right]_{+}}{\sup _{\Omega}\|V\|}\right\} .
$$

The first result of this paper (as given further in Theorem 2.3) gives a weighted version of the Cheeger inequality on a bounded domain in a smooth metric measure space. 
Theorem 2.3 Let $\Omega$ be a bounded domain with smooth boundary, $\partial \Omega \neq \varnothing$, in a complete smooth metric space $(\mathscr{M}, \mathrm{g}, \phi)$. Then, referring to (2.5) and (2.6), we have

$$
\lambda_{1, p}(\Omega) \geq \frac{1}{p^{p}} \mathbf{h}_{\phi}(\Omega)^{p}
$$

Moreover, if $\|\nabla \phi\| \leq c, c \geq 0$, then we have

$$
\lambda_{1, p}(\Omega) \geq \frac{1}{p^{p}} h_{c}(\Omega)^{p} .
$$

Note that when $\phi$ is a constant, we can take $c=0$; then $h=h_{c}$, and $\mathbf{h}_{\phi}$ is the usual Cheeger constant on a Riemannian manifold.

Proof Suppose $\eta \in C_{0}^{\infty}(\Omega)$ is a positive function and let $\mathbf{A}(t):=\{x \in \Omega: \eta(x)>t\}$. Using the coarea formula, we have

$$
\begin{aligned}
\int_{\Omega}|\nabla \eta| e^{-\phi} d v & =\int_{-\infty}^{\infty}\left(\int_{\mathbf{A}(t)} d \mathbf{A}(t)\right) d t=\int_{-\infty}^{\infty} \operatorname{Per}_{\phi}(\mathbf{A}(t)) d t \\
& =\int_{-\infty}^{\infty} \frac{\operatorname{Per}_{\phi}(\mathbf{A}(t))}{\operatorname{Vol}_{\phi}(\mathbf{A}(t))} \operatorname{Vol}_{\phi}(\mathbf{A}(t)) d t \\
& \geq \inf _{\Omega} \frac{\operatorname{Per}_{\phi}(\mathbf{A}(t))}{\operatorname{Vol}_{\phi}(\mathbf{A}(t))} \int_{-\infty}^{\infty} \operatorname{Vol}_{\phi}(\mathbf{A}(t)) d t=\mathbf{h}_{\phi}(\Omega) \int_{\Omega} \eta(x) e^{-\phi} d v
\end{aligned}
$$

For any $p>1$ and $f \in W_{0}^{1, p}(\Omega)$, by the Hölder inequality we have that

$$
\begin{aligned}
\int_{\Omega}\left|\nabla f^{p}\right| e^{-\phi} d v & =p \int_{\Omega}|f|^{p-1}|\nabla f| e^{-\phi} d v \\
& \leq p\left(\int_{\Omega}|f|^{p} e^{-\phi} d v\right)^{\frac{p-1}{p}}\left(\int_{\Omega}|\nabla f|^{p} e^{-\phi} d v\right)^{\frac{1}{p}} .
\end{aligned}
$$

Setting $\eta=f^{p}$, by combining (2.15) and (2.16) we obtain

$$
\mathbf{h}_{\phi}(\Omega) \leq \frac{\int_{\Omega}|\nabla \eta| e^{-\phi} d \nu}{\int_{\Omega} \eta e^{-\phi} d v}=\frac{\int_{\Omega}\left|\nabla f^{p}\right| e^{-\phi} d \nu}{\int_{\Omega}\left|f^{p}\right| e^{-\phi} d \nu} \leq p\left(\frac{\int_{\Omega}|\nabla f|^{p} e^{-\phi} d v}{\int_{\Omega}|f|^{p} e^{-\phi} d \nu}\right)^{\frac{1}{p}}
$$

Since $f \in W_{0}^{1, p}(\Omega)$ is arbitrary, we arrive at

$$
\left(\mathbf{h}_{\phi}(\Omega)\right)^{p} \leq p^{p} \lambda_{1, p}
$$

which concludes the proof of the first part.

Moreover, if $\|\nabla \phi\| \leq c, c \geq 0$, then for any vector field $V \in \mathscr{X}(\Omega)$ and $p>1$, we have the bound

$$
\lambda_{1, p} \geq \frac{1}{p^{p}} \mathbf{h}_{\phi}(\Omega)^{p} \geq \frac{1}{p^{p}} h(\Omega)^{p} \geq \frac{1}{p^{p}} h_{c}(\Omega)^{p} .
$$




\section{Eigenvalue problem for the weighted bi-Laplacian}

In this section, we consider the nonlinear eigenvalue problem involving drifting biLaplacian on compact weighted manifold $(M, \mathrm{~g}, \phi)$, namely, the weighted buckling problem

$$
\begin{cases}\mathscr{L}_{\phi}^{2} f=-\Lambda \mathscr{L}_{\phi} f & \text { in } M, \\ f=\frac{\partial f}{\partial v}=0 & \text { on } \partial M,\end{cases}
$$

where $v$ denotes the outer unit normal vector field to the boundary $\partial M$. By variational characterization we denote the bottom of the spectrum of $-\mathscr{L}_{\phi}^{2}$ for the eigenvalue problem (3.1) by

$$
\Lambda_{1}(M)=\inf _{f}\left\{\frac{\int_{M}\left(\mathscr{L}_{\phi} f\right)^{2} e^{-\phi} d \nu}{\int_{M}\|\nabla f\|^{2} e^{-\phi} d \nu}: f \in W_{0}^{1,2}(M), f \neq 0\right\} .
$$

The spectrum is real and purely discrete, whereas the eigenvalues together with their multiplicities can be arranged in a nondecreasing order

$$
0<\Lambda_{1}(M)<\Lambda_{2}(M) \leq \cdots \nearrow+\infty
$$

\subsection{Escobar-Lichnerowicz-Reilly lower estimates}

We would like to prove the following theorem.

Theorem 3.1 Let $(M, \mathrm{~g}, \phi)$ be an n-dimensional compact smooth metric measure space with smooth boundary $\partial M$ and $\operatorname{Ric}_{\phi}^{m}(M) \geq m \kappa, \kappa \geq 0$. Denote by $\Lambda_{1}(M)$ the first eigenvalue of

$$
\mathscr{L}_{\phi}^{2} f=-\Lambda \mathscr{L}_{\phi} f
$$

on $(M, \mathrm{~g}, \phi)$. If $\partial M \neq \varnothing$, then the boundary conditions

$$
f=\frac{\partial f}{\partial v}=0
$$

hold on $\partial M$. Then

$$
\Lambda_{1}(M) \geq \frac{1}{4} h(M)^{2}+m \kappa,
$$

where

$$
h(M):=\sup _{V}\left\{\frac{\inf _{M}\left[e^{\phi} \operatorname{div}\left(e^{-\phi} V\right)\right]}{\sup _{M}\|V\|}: V \in \Gamma(T M)\right\} .
$$

First, we state some tools (weighted Bochner and Reilly formulas) to prove this theorem.

Weighted Bochner formulas Let $(M, \mathrm{~g}, \phi)$ be an $n$-dimensional compact smooth metric measure space. Then for a $C^{3}$ function $h$ on $M$, we have

$$
\frac{1}{2} \mathscr{L}_{\phi}\left(|\nabla h|^{2}\right)=|\operatorname{Hess} h|^{2}+\left\langle\nabla h, \nabla \mathscr{L}_{\phi} h\right\rangle+\operatorname{Ric}_{\phi}(\nabla h, \nabla h)
$$


and

$$
\frac{1}{2} \mathscr{L}_{\phi}\left(|\nabla h|^{2}\right) \geq \frac{1}{m}\left(\mathscr{L}_{\phi} h\right)^{2}+\left\langle\nabla h, \nabla \mathscr{L}_{\phi} h\right\rangle+\operatorname{Ric}_{\phi}^{m}(\nabla h, \nabla h) .
$$

The proof of (3.4) can be found in [26, 36]. Indeed, the realization of (3.5) from (3.4) is based on the following observation:

By the elementary inequality $(b+d)^{2} \geq \frac{b^{2}}{1+s}-\frac{d^{2}}{s}, s>0$, and by the Cauchy inequality $\mid$ Hess $\left.h\right|^{2} \geq \frac{1}{n}(\Delta h)^{2}$ we obtain

$$
\begin{aligned}
|\operatorname{Hess} h|^{2}+\operatorname{Ric}_{\phi}(\nabla h, \nabla h) & \geq \frac{1}{n}\left(\mathscr{L}_{\phi} h+\langle\nabla \phi, \nabla h\rangle\right)^{2}+\operatorname{Ric}_{\phi}(\nabla h, \nabla h) \\
& \geq \frac{1}{n}\left(\frac{n}{m}\left(\mathscr{L}_{\phi} h\right)^{2}-\frac{n}{m-n}\langle\nabla \phi, \nabla h\rangle^{2}\right)+\operatorname{Ric}_{\phi}(\nabla h, \nabla h) \\
& =\frac{1}{m}\left(\mathscr{L}_{\phi} h\right)^{2}+\operatorname{Ric}_{\phi}^{m}(\nabla h, \nabla h) .
\end{aligned}
$$

For the case of nonempty boundary, the second fundamental form of $\partial M$ for any vector fields $X, Y \in \Gamma(T \partial M)$ is defined by

$$
\mathbb{I}(X, Y)=g\left(\nabla_{X} v, Y\right)
$$

where the mean curvature and weighted mean curvature are respectively defined by

$$
H(x)=\operatorname{tr} \mathbb{I} \quad \text { and } \quad H^{\phi}(x):=H(x)-\langle\nabla \phi, v(x)\rangle .
$$

Now denote by $f_{v}$ the normal derivative of $f$ on $\partial M$ and by $d \sigma$ the weighted $(n-1)$ dimensional Riemannian volume measure on $\partial M$.

Weighted Reilly formula Let $(M, g, \phi)$ be an $n$-dimensional compact smooth metric measure space with smooth boundary. Then

$$
\begin{aligned}
\int_{M}\left(\left(\mathscr{L}_{\phi} h\right)^{2}-\left[|\operatorname{Hess} h|^{2}+\operatorname{Ric}_{\phi}(\nabla h, \nabla h)\right]\right) e^{-\phi} d v \\
=\int_{\partial M}\left(H^{\phi} h_{v}+\mathscr{L}_{\phi, \partial} h\right) h_{\nu} d \sigma+\int_{\partial M}\left(\mathbb{I}\left(\nabla_{\partial} h, \nabla_{\partial} h\right)-\left\langle\nabla_{\partial} h, \nabla_{\partial} h_{v}\right\rangle\right) d \sigma,
\end{aligned}
$$

where $\mathscr{L}_{\phi, \partial} \cdot:=\Delta_{\partial} \cdot-\left\langle\nabla_{\partial} \phi, \nabla_{\partial} \cdot\right\rangle$ and $\nabla_{\partial}$ are the weighted Laplacian and covariant derivative with respect to the induced metric on $\partial M$.

The proof of (3.7) can also be found in [26, 36]. As in the classical case, we can show that both weighted Bochner and Reilly formulas can be used to recover the EscobarLichnerowicz-Reilly lower bound estimates on $\Lambda_{1}(M)$ before we proceed to prove Theorem 3.1, which is the main theorem of this section.

Lemma 3.2 Let $(M, \mathrm{~g}, \phi)$ be an $n$-dimensional compact smooth metric measure space with smooth boundary $\partial M$ and $\operatorname{Ric}_{\phi}^{m}(M) \geq m \kappa, \kappa>0$. Let $\Lambda_{1}(M)$ be the first eigenvalue of $\mathscr{L}_{\phi}^{2} f=$ $-\Lambda \mathscr{L}_{\phi} f$ on $M$. 
1. If $\partial M=\varnothing$, then

$$
\Lambda_{1}(M) \geq \frac{m^{2}}{m-1} \kappa
$$

2. Let $\partial M \neq \varnothing$. For the Dirichlet boundary condition, assume that the weighted mean curvature of $\partial M$ is nonnegative. Then

$$
\Lambda_{1}^{\operatorname{Dir}}(M) \geq \frac{m^{2}}{m-1} \kappa
$$

3. Let $\partial M \neq \varnothing$. For the Neumann boundary condition, assume that the fundamental form of $\partial M$ is nonnegative. Then

$$
\Lambda_{1}^{\mathrm{Neu}}(M) \geq \frac{m^{2}}{m-1} \kappa
$$

Here $\Lambda_{1}^{\mathrm{Dir}}(M)$ and $\Lambda_{1}^{\mathrm{Neu}}(M)$ stand for the first Dirichlet and Neumann eigenvalues, respectively.

Proof (1). Integrating Bochner formula (3.5) with respect to the weighted measure $e^{-\phi} d v$ and using the condition $\operatorname{Ric}_{\phi}^{m}(M) \geq m \kappa, \kappa>0$, we get

$$
0 \geq \frac{1}{m} \int_{M}\left(\mathscr{L}_{\phi} f\right)^{2} e^{-\phi} d v-\int_{M}\left(\mathscr{L}_{\phi} f\right)^{2} e^{-\phi} d v+m \kappa \int_{M}\|\nabla f\|^{2} e^{-\phi} d v
$$

After rearranging, we have

$$
\frac{m-1}{m} \frac{\int_{M}\left(\mathscr{L}_{\phi} f\right)^{2} e^{-\phi} d v}{\int_{M}\|\nabla f\|^{2} e^{-\phi} d v} \geq m \kappa,
$$

which gives the desired result.

Next, we consider the case $\partial M \neq \varnothing$. Combining the Dirichlet boundary condition and $H^{\phi}(x) \geq 0$ (resp., the Neumann boundary condition and $\mathbb{I}(x) \geq 0$ ) with the weighted Reilly formula (3.7) and the lower bound for the tensor $\operatorname{Ric}_{\phi}^{m}(M) \geq m \kappa, \kappa>0$, yields the desired estimate for (2) (resp., (3)).

Proof of Theorem 3.1 Applying the Bochner formula (3.4) for the case $\partial M=\varnothing$ and weighted Reilly formula (3.7) for the case $\partial M \neq \varnothing$, combined with boundary conditions $f=f_{v}=0$ on $\partial M$, we have

$$
\int_{M}\left(\mathscr{L}_{\phi} f\right)^{2} e^{-\phi} d v \geq \int_{M}\left[|\operatorname{Hess} f|^{2}+m \kappa\|\nabla f\|^{2}\right] e^{-\phi} d v
$$

which implies

$$
\frac{\int_{M}\left(\mathscr{L}_{\phi} f\right)^{2} e^{-\phi} d \nu}{\int_{M}\|\nabla f\|^{2} e^{-\phi} d \nu} \geq \frac{\int_{M}|\operatorname{Hess} f|^{2} e^{-\phi} d \nu}{\int_{M}\|\nabla f\|^{2} e^{-\phi} d \nu}+m \kappa .
$$

Here we have used the boundary condition, nonnegativity condition on the fundamental form, or weighted mean curvature of the boundary when it is nonempty. 
Now for any $f \in C_{0}^{\infty}(M)$, the vector field $\|\nabla f\|^{2} V$ has a compact support on $M$. Thus a straightforward computation shows that

$$
\begin{aligned}
\operatorname{div}\left(\|\nabla f\|^{2} V e^{-\phi}\right) & =2\|\nabla f\| \nabla|\nabla f| V e^{-\phi}+\|\nabla f\|^{2} \operatorname{div}\left(V e^{-\phi}\right) \\
& \geq\left(-2\|\nabla f\||\nabla| \nabla f||\|V\|+\inf _{M}\left[e^{\phi} \operatorname{div}\left(V e^{-\phi}\right)\right]\|\nabla f\|^{2}\right) e^{-\phi} .
\end{aligned}
$$

By the Young inequality it follows for $\epsilon>0$ that

$$
-2\|\nabla f\||\nabla| \nabla f||\|V\| \geq-2\left(\frac{\mid \nabla \nabla f \|^{2}}{2 \epsilon^{2}}+\frac{\epsilon^{2}\|\nabla f\|^{2}\|V\|^{2}}{2}\right) .
$$

Note that the divergence theorem implies

$$
\int_{M} e^{\phi} \operatorname{div}\left(\|\nabla f\|^{2} V e^{-\phi}\right) e^{-\phi} d v=\int_{\partial M}\|\nabla f\|^{2}\langle V, v\rangle d \sigma=0
$$

since $f=f_{v}=0$ on $\partial M$ implies $\left.(\nabla f)\right|_{\partial M}=0$. In the case of empty boundary, $\int_{M} e^{\phi} \operatorname{div}\left(|\nabla f|^{2} V e^{-\phi}\right) e^{-\phi}=0$ also holds. Now combining (3.9) and (3.10) with the divergence theorem, we have

$$
0 \geq \int_{M} \inf _{M}\left[e^{\phi} \operatorname{div}\left(V e^{-\phi}\right)\right]\|\nabla f\|^{2} e^{-\phi} d \nu-\frac{1}{\epsilon^{2}} \int_{M}\left[\left.|\nabla| \nabla f\right|^{2}+\epsilon^{4}\|\nabla f\|^{2}\|V\|^{2}\right] e^{-\phi} d \nu,
$$

and thus

$$
\left.\int_{M}|\nabla| \nabla f\right|^{2} e^{-\phi} d v \geq \epsilon^{2}\left(\inf _{M}\left[e^{\phi} \operatorname{div}\left(V e^{-\phi}\right)\right]-\epsilon^{2} \sup _{M}\|V\|^{2}\right) \int_{M}\|\nabla f\|^{2} e^{-\phi} d v .
$$

Following the idea of the maximization procedure (see [6]), we know that

$$
\max _{\epsilon>0}\left\{\epsilon^{2}\left(\inf _{M}\left[e^{\phi} \operatorname{div}\left(V e^{-\phi}\right)\right]-\epsilon^{2} \sup _{M}\|V\|^{2}\right)\right\}=\left(\frac{\inf _{M}\left[e^{\phi} \operatorname{div}\left(V e^{-\phi}\right)\right]}{2 \sup _{M}\|V\|}\right)^{2} .
$$

Substituting (3.13) into inequality (3.12), we have

$$
\frac{\int_{M}|\nabla| \nabla f \|^{2} e^{-\phi} d v}{\int_{M}\|\nabla f\|^{2} e^{-\phi} d \nu} \geq\left(\frac{\inf _{M}\left[e^{\phi} \operatorname{div}\left(V e^{-\phi}\right)\right]}{2 \sup _{M}\|V\|}\right)^{2}
$$

and then taking the supremum on the right-hand side over all vector fields $V \in \Gamma(T M)$ gives

$$
\frac{\int_{M}|\nabla| \nabla f \|^{2} e^{-\phi} d v}{\int_{M}\|\nabla f\|^{2} e^{-\phi} d v} \geq\left(\sup _{V} \frac{\inf _{M}\left[e^{\phi} \operatorname{div}\left(V e^{-\phi}\right)\right]}{2 \sup _{M}\|V\|}\right)^{2}
$$

Now we note that

$$
|\nabla| \nabla f||^{2} \leq|\operatorname{Hess} f|^{2}
$$


on $M$ for any $f \in W_{0}^{1,2}$ by the classical form of Kato inequality [13]. Therefore, combining (3.8), (3.14), and (3.15), it follows that

$$
\frac{\int_{M}\left(\mathscr{L}_{\phi} f\right)^{2} e^{-\phi} d \nu}{\int_{M}\|\nabla f\|^{2} e^{-\phi} d \nu} \geq \frac{1}{4} \sup _{V}\left(\frac{\inf _{M}\left[e^{\phi} \operatorname{div}\left(V e^{-\phi}\right)\right]}{\sup _{M}\|V\|}\right)^{2}+m \kappa .
$$

This completes the proof of Theorem 3.1.

Remarks The strategy adopted in the proof of Theorem 3.1 is coined from the idea introduced by Cheung and Leung [18] for submanifolds with bounded mean curvature in the hyperbolic space. (See also Bessa and Montenegro [11, 12]). Note that Cheung-Leung's result [18] can be regarded as an extended version of McKean lower bound [28]. McKean's lower bound for weighted $p$-fundamental tone has been recently studied by the first author on a complete noncompact simply connected smooth metric measure space with sectional curvature bounded from above by negative constant $[2,6]$.

Theorem 3.1 extends the result of Zhang and Zhao [38, Theorem 3.1], where they considered the first eigenvalue $\lambda_{1}(N)$ for buckling problem on an $n$-dimensional compact connected Riemannian manifold $N$ with smooth boundary and $\operatorname{Ric}(N) \geq K>0$ and obtained

$$
\lambda_{1}(N) \geq\left(\frac{\inf _{N} \operatorname{div}(V)}{2\|V\|_{\infty}}\right)^{2}+K
$$

for any vector $V$ satisfying $\inf _{N} \operatorname{div}(V)>0$. Clearly, (3.16) coincides with (3.3) if and only if $\phi$ is constant.

\subsection{Estimates on weighted geodesic balls}

We apply Theorem 3.1 to give lower bounds for the first eigenvalue on the drifting biLaplacian on a geodesic ball whose radius does not exceed the injectivity radius. First, we state an important lemma on Hessian comparison (see [16] for details), which will help realize the desired estimates. This idea has been applied previously by several authors $[6,11,19,38]$ to different settings.

Lemma 3.3 (Hessian comparison theorem) Let $(\mathscr{M}, \mathrm{g})$ be a complete Riemannian manifold. Consider points $x_{0}, x_{1} \in M$ and a minimizing geodesic $\gamma:\left[0, \rho\left(x_{1}\right)\right] \rightarrow M$ joining $x_{0}$ and $x_{1}$ with $\rho(x)$ being the distance function measured from the base point $x_{0}$. Define the function

$$
\mu(\rho)= \begin{cases}k \operatorname{coth}(k \rho) & \text { if } \sup _{\gamma} \mathscr{K}_{M}=-k^{2}, \\ 1 / \rho & \text { if } \sup _{\gamma} \mathscr{K}_{M}=0, \\ k \cot (k \rho) & \text { if } \sup _{\gamma} \mathscr{K}_{M}=+k^{2} \text { and } \rho<\frac{\pi}{2 k},\end{cases}
$$

where $\mathscr{K}_{M}$ denotes the sectional curvature of $M$. Then the Hessians of $\rho$ and $\rho^{2}$ at the point $x$ satisfy the following estimates:

$$
\begin{aligned}
& \nabla^{2} \rho(x)(Y, Y) \geq \mu(\rho(Y))\|Y\|^{2}, \quad \nabla^{2} \rho(x)\left(\gamma^{\prime}, \gamma^{\prime}\right)=0, \\
& \nabla^{2} \rho^{2}(x)(Y, Y) \geq 2 \rho(x) \mu(\rho(x))\|Y\|^{2}, \quad \nabla^{2} \rho^{2}(x)\left(\gamma^{\prime}, \gamma^{\prime}\right)=2,
\end{aligned}
$$

where $Y$ is any vector in $T_{x} M$ perpendicular to $\gamma^{\prime}(\rho(x))$. 
From (3.18) the following lower bounds with respect to Laplacian for the distance function $\rho$ and squared distance function $\rho^{2}$ can be deduced (see $[6,11,38]$ ):

$$
\begin{aligned}
& \Delta \rho(x) \geq(m-1) \mu(\rho(x)), \\
& \Delta \rho^{2}(x) \geq 2(m-1) \rho(x) \mu(\rho(x))+2 .
\end{aligned}
$$

Let $\mathscr{B}_{r}(x)$ be a geodesic ball centered at $x$ with radius $r$ in $(M, g, \phi)$. By the variational characterization discussed above we have

$$
\Lambda_{1}\left(\mathscr{B}_{r}(x)\right)=\inf _{f \in H^{2}\left(\mathscr{B}_{r}\right), f \neq 0} \frac{\int_{\mathscr{B}_{r}}\left(\mathscr{L}_{\phi} f\right)^{2} e^{-\phi} d v}{\int_{\mathscr{B}_{r}}\|\nabla f\|^{2} e^{-\phi} d v}
$$

Theorem 3.4 Let $(M, \mathrm{~g}, \phi)$ be a smooth metric measure space with potential function satisfying $\|\nabla \phi\| \leq$ for some $c \geq 0$. Let $\mathscr{B}_{r}(q)$ denote the geodesic ball centered at $q$ with radius $r<\operatorname{inj}(q)$, and let $k_{r}(q)=\sup \left\{\mathscr{K}_{M}(x): x \in \mathscr{B}_{r}(q)\right\}$, where $\mathscr{K}_{M}(x)$ denotes the sectional curvature of $M$ at $x$, and $\operatorname{Ric}_{\phi}^{m}\left(\mathscr{B}_{r}\right) \geq m \kappa, \kappa \geq 0$. Then

$$
\Lambda_{1}\left(\mathscr{B}_{r}(q)\right) \geq \begin{cases}{[m-c r]_{+}^{2} / 4 r^{2}+m \kappa,} & k_{r}(q)=0, \\ {[(m-1) k r \cot (k r)+1-c r]_{+}^{2} / 4 r^{2}+m \kappa,} & k_{r}(q)=+k^{2}, r<\frac{\pi}{2 k},\end{cases}
$$

where $k$ is a nonzero constant.

Proof Since $\rho^{2}$ is a smooth function in $\mathscr{B}_{r}$, we can set $V=\nabla \rho^{2}$. Then $\sup _{\mathscr{B}_{r}}\|V\|=2 r$, and

$$
\begin{aligned}
\inf _{\mathscr{B}_{r}}\left[e^{\phi} \operatorname{div}\left(e^{-\phi} V\right)\right] & =\inf _{\mathscr{B}_{r}} \mathscr{L}_{\phi} \rho^{2}=\inf _{\mathscr{B}_{r}}\left[\Delta \rho^{2}-\left\langle\nabla \phi, \nabla \rho^{2}\right\rangle\right] \\
& \geq \inf _{\mathscr{B}_{r}} \Delta \rho^{2}-c \sup _{\mathscr{B}_{r}}\|V\| \geq 2\left[\inf _{\mathscr{B}_{r}}[(m-1) \rho \mu(\rho)+1]-c r\right],
\end{aligned}
$$

where we have used the second inequality in (3.19). Hence by Theorem 3.1 we have

$$
\begin{aligned}
\Lambda_{1}\left(\mathscr{B}_{r}\right) & \geq \frac{1}{4} h\left(\mathcal{B}_{r}\right)^{2}+m \kappa \\
& \geq \frac{1}{4}\left(\sup _{V}\left\{\frac{\inf _{\mathscr{B}_{r}}\left[e^{\phi} \operatorname{div}\left(e^{-\phi} V\right)\right]}{\sup _{\mathscr{B}_{r}}\|V\|}\right\}\right)^{2}+m \kappa \\
& \geq \frac{1}{4}\left(\frac{1}{r} \inf _{\mathscr{B}_{r}}[(m-1) \rho \mu(\rho)+1]-c\right)^{2}+m \kappa,
\end{aligned}
$$

where $V \in \mathscr{X}\left(\mathscr{B}_{r}\right)$. This leads to the desired conclusion since $k_{r}(q) \geq 0$. Specifically, for $k_{r}(q)=0, \mu(\rho)=1 / \rho$, and then

$$
\Lambda_{1}\left(\mathscr{B}_{r}\right) \geq \frac{1}{4}\left(\frac{(m-1)+1-c r}{r}\right)^{2}+m \kappa=\frac{1}{4}\left(\frac{m-c r}{r}\right)^{2}+m \kappa .
$$

\subsection{Estimates on submanifolds with bounded mean curvaure}

Theorem 3.5 Let $M=(M, \mathrm{~g}, \phi)$ be a smooth metric measure space with potential function satisfying $\|\nabla \phi\| \leq c$, where $c \geq 0$. Let $\vartheta: M \hookrightarrow N$ be an isometric immersion with locally 
bounded weighted mean curvature, that is, the number $\hbar(q, r):=\sup \left\{\left|H^{\phi}(x)\right|: x \in \vartheta(M) \cap\right.$ $\left.\mathscr{B}_{r}^{N}(q), q \in N \backslash \vartheta(M), r>0\right\}$ is finite, where $\mathscr{B}_{r}^{N}(q)$ denotes the geodesic ball centered at $q$ with radius $r<\operatorname{inj}(q)$ in $N$. Suppose $\Omega_{\phi}$ is any connected component of $\vartheta^{-1}\left(\overline{\mathscr{B}_{r}^{N}(q)}\right)$. Let $k_{r}(q)=\sup \left\{\mathscr{K}_{M}(x): x \in \mathscr{B}_{r}^{M}(q)\right\}$, where $\mathscr{K}_{M}(x)$ is the sectional curvature of $M$ in $\mathscr{B}_{r}^{M}(q)$, and $\operatorname{Ric}_{\phi}^{m}\left(\Omega_{\phi}\right) \geq m \kappa, \kappa \geq 0$. Then:

1. If $k_{\mathrm{inj}(q)}(q)=k^{2}<+\infty, \operatorname{Ric}_{\phi}^{m}\left(\Omega_{\phi}\right) \geq m \kappa, \kappa \geq 0$, and $r<\min \left\{\operatorname{inj}(q), \frac{\pi}{2 k}, \frac{1}{k} \cot ^{-1}\left(\frac{\hbar(q, \operatorname{inj}(q))}{(m-1) k}\right)\right\}$, then

$$
\Lambda_{1}\left(\Omega_{\phi}\right) \geq[(m-1) k \cot (k r)-\hbar(q, r)-c]_{+}^{2} / 4+m \kappa .
$$

2. If $k_{r}(q)>0, \forall r>0, \lim _{r \rightarrow \infty} k_{r}(q)=\infty$, $\operatorname{inj}(q)=\infty, \operatorname{Ric}_{\phi}^{m}\left(\Omega_{\phi}\right) \geq m \kappa, \kappa \geq 0$, and $r(s)=\min \left\{\frac{\pi}{2} \sqrt{k_{s}(q)}, \frac{1}{k_{s}(q)} \cot ^{-1}\left(\frac{\hbar(q, s)}{(m-1) k_{s}(q)}\right)\right\}$, then

$$
\Lambda_{1}\left(\Omega_{\phi}\right) \geq\left[(m-1) \sqrt{k_{r}(q)} \cot \left(r \sqrt{k_{r}(q)}\right)-\hbar(q, r)-c\right]_{+}^{2} / 4+m \kappa .
$$

3. If $k_{\mathrm{inj}(q)}(q)=0, \operatorname{Ric}_{\phi}^{m}\left(\Omega_{\phi}\right) \geq m \kappa, \kappa \geq 0$, and $r<\min \left\{\operatorname{inj}(q), \frac{m}{\hbar(q, \operatorname{inj}(q))}\right\}$, where $\frac{m}{\hbar(q, \operatorname{inj}(q))}=+\infty$ when $\hbar(q, \operatorname{inj}(q))=0$, then

$$
\Lambda_{1}\left(\Omega_{\phi}\right) \geq[m-\hbar(q, r) r-c r]_{+}^{2} / 4 r^{2}+m \kappa .
$$

Note that the weighted mean curvature $H^{\phi}(x)$ appearing in Theorem 3.5 is defined in a similar way to definition in (3.6), though not restricted only to the boundary. We thus retain the same notation since it is clear from the context used here.

Proof Let $\rho(x)$ be the distance function on $N$, and let $f_{j}=\rho^{j} \circ \vartheta$ on $M, j=1,2$. Recall that $\Omega_{\phi}$ is a connected component of $\vartheta^{-1}\left(\overline{\mathscr{B}_{r}^{N}(q)}\right)$ and $f_{j}$ are smooth on $\vartheta^{-1}\left(\mathscr{B}_{r}^{N}(q)\right)$. Denoting $V_{j}=\nabla f_{j}, j=1,2$, and using (2.11), we obtain

$$
\inf _{\Omega_{\phi}}\left[e^{\phi} \operatorname{div}\left(e^{-\phi} V_{1}\right)\right] \geq\left[\inf _{\Omega_{\phi}} \operatorname{div}\left(V_{1}\right)-c \sup _{\Omega_{\phi}}\left\|V_{1}\right\|\right]_{+} .
$$

(1) If $k_{r}(q)=k^{2}$ and $r<\min \left\{\operatorname{inj}(q), \frac{\pi}{2 k}, \frac{1}{k} \cot ^{-1}\left(\frac{\hbar(q, \operatorname{inj}(q))}{(m-1) k}\right)\right\}=\min \left\{\operatorname{inj}(q), \frac{1}{k} \cot ^{-1}\left(\frac{\hbar(q, \operatorname{inj}(q))}{(m-1) k}\right)\right\}$, then by (3.21) we have

$$
\inf _{\Omega_{\phi}}\left[e^{\phi} \operatorname{div}\left(e^{-\phi} V_{1}\right)\right] \geq\left[(m-1) k \cot (k r)-\hbar(q, r)-c \sup _{\Omega_{\phi}}\left\|V_{1}\right\|\right]_{+} .
$$

(2) If the hypotheses of (2) hold, analogously to (1), we have

$$
\inf _{\Omega_{\phi}}\left[e^{\phi} \operatorname{div}\left(e^{-\phi} V_{1}\right)\right] \geq\left[(m-1) \sqrt{k_{r}(q)} \cot \left(r \sqrt{k_{r}(q)}\right)-\hbar(q, r)-c \sup _{\Omega_{\phi}}\left\|V_{1}\right\|\right]_{+} .
$$

(3) If $k_{r}(q)=0$ and $r<\min \left\{\operatorname{inj}(q), \frac{m}{\hbar(q, \operatorname{inj}(q))}\right\}$, then using (2.11), we obtain

$$
\begin{aligned}
\inf _{\Omega_{\phi}}\left[e^{\phi} \operatorname{div}\left(e^{-\phi} V_{2}\right)\right] & \geq \inf _{\Omega_{\phi}} \operatorname{div}\left(V_{2}\right)-c \sup _{\Omega_{\phi}}\left\|V_{2}\right\| \\
& \geq\left[2(m-\hbar(q, r) r)-c \sup _{\Omega_{\phi}}\left\|V_{2}\right\|\right]_{+} .
\end{aligned}
$$


Since $\left\|V_{1}\right\| \leq 1$ and $\left\|V_{2}\right\| \leq 2 r$, the desired estimate in each case follows by Theorem 3.1.

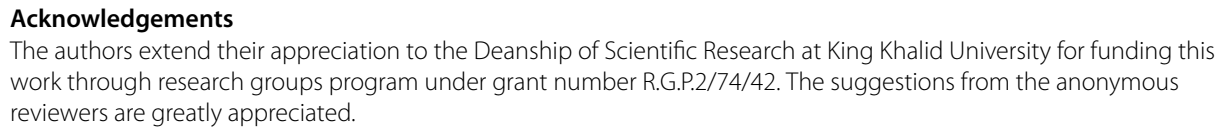

Availability of data and materials

Not applicable.

\section{Competing interests}

The authors declare that they have no competing interests.

\section{Authors' contributions}

The main idea of this paper was proposed by A. Abolarinwa. All authors read and approved the final manuscript. All authors contributed equally to the writing of this paper.

\section{Author details}

'Department of Mathematics, University of Lagos, Akoka, Lagos State, Nigeria. ${ }^{2}$ Department of Mathematics, College of Science, King Khalid University, 9004, Abha, Saudi Arabia.

\section{Publisher's Note}

Springer Nature remains neutral with regard to jurisdictional claims in published maps and institutional affiliations.

Received: 10 February 2021 Accepted: 24 May 2021 Published online: 31 May 2021

\section{References}

1. Abolarinwa, A.: The first eigenvalue of $p$-Laplacian and geometric estimates. Nonl. Anal. Diff. Eq. 2, $105-115$ (2014)

2. Abolarinwa, A.: Lower estimates on the principal eigenvalue of Witten $q$-Laplacian on smooth metric measure spaces. Nig. J. Math. Appl. 30, 96-110 (2020)

3. Abolarinwa, A., Adebimpe, O., Bakare, E.A.: Monotonicity formulas for the first eigenvalue of the weighted $p$-Laplacian under the Ricci-harmonic flow. J. Inequal. Appl. 2019, 10 (2019)

4. Abolarinwa, A., Azami, S.: Comparison estimates on the first eigenvalue of a quasilinear elliptic system. J. Appl. Anal. 26(2), 273-285 (2020)

5. Abolarinwa, A., Edeki, S.O., Ehigie, J.O.: On the spectrum of the weighted p-Laplacian under the Ricci-harmonic flow. J. Inequal. Appl. 2020, 58 (2020)

6. Abolarinwa, A., Taheri, A.: Geometric estimates on weighted $p$-fundamental tone and applications to the first eigenvalue of submanifolds with bounded mean curvature. Complex Var. Elliptic Equ. (2021). https://doi.org/10.1080/17476933.2021.1873961

7. Abolarinwa, A., Yang, C., Zhang, D.: On the spectrum of the $p$-biharmonic operator under the Ricci flow. Results Math. 54, 1-16 (2020)

8. Bakry, D., Émery, M.: Diffusions hypercontractives. In: Azḿa, J., Yor, M. (eds.) Séminaire de Probabilités XIX 1983/84 LNM, vol. 1123. Springer, Berlin (1985)

9. Bakry, D., Gentil, I., Ledoux, M.: Analysis and Geometry of Markov Diffusion Operators. A Series of Comprehensive Studies in Mathematics, vol. 348. Springer, Berlin (2012)

10. Benson, B.: The Cheeger constant, isoperimetric problems, and hyperbolic surfaces. arXiv:1509.08993

11. Bessa, G.P., Montenegro, J.F.: Eigenvalue estimates for submanifolds with locally bounded mean curvature. Ann. Glob. Anal. Geom. 24, 279-290 (2003)

12. Bessa, G.P., Montenegro, J.F.: An extension of Barta's theorem and geometric applications. Ann. Glob. Anal. Geom. 31, 345-362 (2007)

13. Calderbank, D.M.J., Gauduchon, P., Herzlich, M.: On the Kato inequality in Riemannian geometry. Semin. Congr. 4, 95-113 (2000)

14. Chavel, I.: Eigenvalues in Riemannian Geometry. Academic Press, San Diego (1984)

15. Cheeger, J.: A lower bound for the smallest eigenvalue of the Laplacian. In: Problems in Analysis (Papers dedicated to Salomon Bochner, 1969), pp. 195-7199. Princeton Univ. Press, Princeton (1970)

16. Cheeger, J., Ebin, D.G.: Comparison Theorems in Riemannian Geometry. Am. Math. Soc., Providence (1975)

17. Chen, D., Chen, Q.-M., Wang, Q., Xia, C.: On eigenvalue of a system of elliptic equations and of the biharmonic operator. J. Math. Anal. Appl. 387, 1146-1159 (2012)

18. Cheung, L.F., Leung, P.F.: Eigenvalue estimates for submanifolds with bounded mean curvature in the hypaerbolic space. Math. Z. 236, 525-530 (2001)

19. Evangelista, I., Seo, K.: p-Fundamental tone estimates of submanifolds with bounded mean curvature. Ann. Glob. Anal. Geom. 52, 269-287 (2017)

20. Futaki, A., Li, H., Li, X.D.: On the first eigenvalue of the Witten-Laplacian and the diameter of compact shrinking solitons. Ann. Glob. Anal. Geom. 44, 105-114 (2013) 
21. Grigor'yan, A.: Heat Kernel and Analysis on Manifolds. AMS/IP, Providence (2013)

22. Hamilton, R.S.: The Ricci flow on surfaces. Contemp. Math. 71, 237-261 (1988)

23. Lambert, C., Suneeta, V.: Stability analysis of the Witten black hole (cigar soliton) under world-sheet renormalization group flow. Phys. Rev. D 86(8), 084041 (2012)

24. Li, X., Xiong, X., Zeng, L.: Estimates for the eigenvalues of the bi-drifting Laplacian on cigar soliton. J. Geom. Phys. 61(10), $101404(2020)$

25. Lima, B.P., Montenegro, J.F., Santos, N.L.: Eigenvalues estimates for the p-Laplace operator on manifolds. Nonlinear Anal. 72, 771-781 (2010)

26. Ma, L., Du, S.-H.: Extension of Reilly formula with applications to eigenvalue estimates for drifting Laplacians. C. R. Acad. Sci. Paris, Ser. I 1348, 1023-1206 (2010)

27. Matei, A.M.: First eigenvalue of the $p$-Laplace operator. Nonlinear Anal. 39, 1051-1068 (2000)

28. McKean, H.P.: An upper bound to the spectrum of $\Delta$ on a manifold of negative curvature. J. Differ. Geom. 4, 359-366 (1970)

29. Munteanu, O., Wang, J.: Smooth metric measure spaces with nonnegative curvature. Commun. Anal. Geom. 19, 451-486 (2011)

30. Munteanu, O., Wang, J.: Analysis of weighted Laplacian and applications to Ricci solitons. Commun. Anal. Geom. 20, 55-94 (2012)

31. Parini, E.: An introduction to the Cheeger problem. Surv. Math. Appl. 6, 9-22 (2011)

32. Perelman, G.: The entropy formula for the Ricci flow and its geometric application (2002). arXiv:math.DG/0211159v1

33. Schoen, R., Yau, S.T.: Lectures on Differential Geometry. International Press, Somerville (1994)

34. Shi, Y.M., Zhang, H.C.: Lower bounds for the first eigenvalue on compact manifolds. Chin. Ann. Math., Ser. A 28(6), 863-866 (2007)

35. Wang, L.F.: Eigenvalue estimate for the weighted p-Laplacian. Ann. Math. 191, 539-550 (2012)

36. Wang, Y.-Z., Li, H.-Q.: Lower bound estimates for the first eigenvalue of the weighted $p$-Laplacian on smooth metric measure spaces. Differ. Geom. Appl. 45, 23-42 (2016)

37. Yau, S.T.: Isoperimetric constant and the first eigenvalue of a compact Riemannian manifold. Ann. Sci. Éc. Norm. Supér. 8, 487-507 (1975)

38. Zang, L., Zhao, Y.: The lower bounds of the first eigenvalue for the biharmonic operator on manifolds. J. Inequal. Appl. 2016, 5 (2016)

39. Zeng, L.: Estimates for the eigenvalues of the bi-drifting Laplacian on complete metric measure spaces. J. Math. Phys. 61, $101504(2020)$

40. Zeng, L., Zhu, H.: Eigenvalues of Witten-Laplacian on the cigar metric measure spaces. Anal. Math. Phys. 11, 41-78 (2021)

\section{Submit your manuscript to a SpringerOpen ${ }^{\circ}$ journal and benefit from:}

- Convenient online submission

- Rigorous peer review

- Open access: articles freely available online

- High visibility within the field

- Retaining the copyright to your article

Submit your next manuscript at $>$ springeropen.com 